\title{
Efficiency of Nicotinamide-based Supportive Therapy in Lymphopenia for Patients With COVID-19: A Randomized Controlled Trial
}

\section{Qiang Hu}

General Hospital of Northern Theater Command

Quan-Yu Zhang

General Hospital of Northern Theater Command

Cheng-Fei Peng

General Hospital of Northern Theater Command

Zhuang Ma

General Hospital of Northern Theater Command

Ya-Ling Han ( $\sim$ hanyaling@163.net)

General Hospital of Northern Theater Command

\section{Research Article}

Keywords: niacinamide, COVID-19, lymphopenia, cytokine storm

Posted Date: January 11th, 2022

DOI: https://doi.org/10.21203/rs.3.rs-1173313/v1

License: (c) (i) This work is licensed under a Creative Commons Attribution 4.0 International License.

Read Full License 


\section{Abstract}

Background: The purpose of this study was to investigate the efficiency of nicotinamide-based supportive therapy in lymphopenia for patients with coronavirus disease-2019 (COVID-19).

Methods: 24 patients diagnosed with the COVID-19 were randomly divided into two groups $(n=12)$ during hospitalization in the ratio of $1: 1$. Based on the conventional treatment, the treatment group was given $100 \mathrm{mg}$ nicotinamide, five times a day. The control group only received routine treatments. The primary endpoint was the change in absolute lymphocyte counts. The secondary endpoints included both the inhospital death and the composite endpoint of aggravation, according to upgraded oxygen therapy, improvement of nursing level, and ward rounds of superior physicians for changes of conditions.

Results: The full blood counts before and after receiving the nicotinamide were comparable in each group (all $\mathrm{P}>0.05$ ). Before and after receiving the nicotinamide, mean absolute lymphocyte counts were similar between the two groups $\left([0.94 \pm 0.26] * 10^{9} / L\right.$ versus $[0.89 \pm 0.19] * 10^{9} / L, P=0.565 ;[1.15 \pm 0.48]^{*} 10^{9} / L$ versus $[1.02 \pm 0.28]^{*} 10^{9} / \mathrm{L}, \mathrm{P}=0.445$, respectively). Therefore, there was no statistically significant difference in the lymphocyte improvement rate between the two groups $(23.08 \pm 46.10$ versus $16.52 \pm 24.10, P=0.67)$. There was also no statistically significant difference for the secondary endpoints between the two groups.

Conclusion: Among patients with COVID-19, there was no statistically significant difference in change of full blood counts and the absolute lymphocyte counts before and after intervention in both groups. Therefore, no new evidence was found for the effect of niacinamide on lymphopenia in patients with COVID-19.

Trial registration: ClinicalTrials.gov, NCT04910230. Registered 1 June 2021-retrospectively registered.

\section{Background}

The Severe Acute Respiratory Syndrome Corona Virus 2 (SARS-CoV-2) has spread around the world rapidly, with high lethality[1]. Several studies have shown the patients with coronavirus disease 2019 (COVID-19) in severe conditions may have many abnormal laboratory parameters such as lactate dehydrogenase, Ddimer, C-reactive protein, and pro-inflammatory cytokines[2-6]. Similarly, there is a decrease in lymphocyte counts at an early stage in patients with COVID-19 especially in severe cases[4-7]. Previous studies reported that the percentage of peripheral blood lymphocyte count gradually decreased and remained at a low level during the devastation of COVID-19[8]. On the contrary, the improvement of lymphocyte counts from a low level indicates a relatively good prognosis. Therefore, early treatment to improve the lymphocyte counts of patients with COVID-19 can theoretically prevent further deterioration and decrease the mortality rate, which can finally improve their prognosis.

Lymphopenia is one of the most prominent characteristics of COVID-19 which is in contrast to other viral infections. Although the definite mechanism is unclear, recent studies suggested NAD+ consumption may be involved in this process[9]. NAD+, known as the central catalyst of metabolism, has been shown 
connected to several stages of defense against SARS-CoV-2 infection and works like the fuel that supports immune defense. Upon activation of the innate immune response, poly-ADP-ribose polymerases (PARPs) hyperactivated due to virus entry leads to NAD+ depletion and cell death[10]. During the adaptive immune response, the overexpression of CD38 activity in both CD4+ and CD8+ lymphocytes highly correlates with the depletion of NAD+, which leads to increased pro-inflammatory cytokines[11]. However, few studies have demonstrated the effectiveness of niacinamide on lymphopenia in COVID-19 patients. Based on the above research results, we intended to explore whether replenishing nicotinamide, a precursor of NAD+ can be used as supportive therapy for treating lymphopenia in patients with COVID-19.

\section{Methods}

\section{Study design}

This study was a single-center, randomized, open-label clinical trial designed to examine the effectiveness of nicotinamide-based supportive therapy in lymphopenia for patients with COVID-19. Patients diagnosed as the COVID-19, who were admitted to Huo Shen Shan Hospital from March 1, 2020, to April 2, 2020, were eligible for participation, as shown in Fig.1. Our study was approved by the National Health Commission of China, the institutional review board at Huo Shen Shan Hospital, and the institutional ethics committee of the General Hospital of Northern Theater Command of the Chinese People's Liberation Army, and the study was in accordance with Helsinki Declaration. The trial was registered with ClinicalTrials.gov, number NCT04910230. All participants provided written informed consent. An independent Data Safety Monitoring Board (DSMB) would review the unblinded data in each group regularly and make suggestions to the sponsors if our study had warranted early termination. Authors declared our study adhere to the CONSORT guideline.

\section{Patients}

For inclusion criteria in the study, patients aged 18 to 85 , who were diagnosed as COVID-19 were included in our study. The exclusion criteria included: 1) the mild or critically ill patients, 2) participation in other clinical trials, 3) pregnancy or lactation, 3) ALT/AST > 5 times the upper limit of normal (ULN), neutrophils counts $<0.5^{*} 10^{9} / \mathrm{L}$, platelets counts $<50 * 10^{9} / \mathrm{L}$, 4) patients diagnosed with rheumatoid immune-related diseases, 5) long-term use of oral immunosuppressive drugs or immunomodulatory drugs, 6) hypersensitive reaction to nicotinamide or any auxiliary materials, 7) comorbidities including active tuberculosis or bacterial or fungal infections, 8) undergone an organ transplant, 9) mental disorders. The diagnosis and classification of COVID-19 were based on the "Guideline for the Diagnosis and Treatment of Novel Coronavirus (2019-nCoV) infection" published by the National Health Commission of China[12]. The mild type was defined as patients with minor symptoms but no manifestation of pneumonia on imaging. The ordinary type was defined as patients having fever and respiratory symptoms with a positive image of pneumonia. The severe type was defined as patients with any of the following characteristics: respiratory distress (respiratory rate $>30$ times per minute), oxygen saturation $\mathrm{Sp} 02 \leq 93 \%$, partial arterial oxygen pressure $(\mathrm{PaO} 2) /$ fraction of inspired oxygen $(\mathrm{FiO} 2) \leq 300 \mathrm{mmHg}$. Patients with critically ill were identified if 
they had one of the following features: respiratory failure requiring mechanical ventilation, shock, organ dysfunction that needs ICU admission.

\section{Randomization and treatment}

Eligible patients were recruited for participation when the first occurrence of the absolute lymphocyte level under the $1.1 * 10^{9} / \mathrm{L}$ during hospitalization. Enrolled participants were randomly assigned $(1: 1)$ into the intervention group receiving nicotinamide treatment or the control group with only routine treatments. $A$ random number table and opaque, sealed envelopes marked with the sequence number were used to conduct the randomization. The treatment group received $100 \mathrm{mg}$ of nicotinamide (Shanghai Xinyi Pharmaceutical Co. Ltd.) five times a day. Other routine treatments in the intervention group and control group were prescribed at physicians' discretion.

\section{Data collection and clinical assessment}

Baseline characteristics including the demographic description, diagnosis, comorbidities, and medication were collected via the electronic medical record system. Blood tests were done before and 48 hours after intervention in all participants. The full blood counts in both groups were assessed before and $48 \mathrm{~h}$ after treatment. As a result, we would have absolute lymphocyte counts before and after the intervention both in the two groups. Meanwhile, we would compare the changes of the absolute changes of lymphocyte counts and lymphocyte improvement rates between the two groups. Moreover, the secondary endpoints were recorded accordingly to compare the incidence of events in the two groups.

\section{Endpoints}

The primary endpoint was the change of absolute lymphocyte counts between the baseline and the 48hour blood tests. The secondary endpoints included in-hospital death and the composite endpoint of upgraded oxygen therapy, improvement of nursing level, and ward rounds of superior physicians for changes of conditions. The upgraded oxygen therapy was defined as the upgrading of general highvolume oxygen intake from oxygen intake, ventilator using from high-volume oxygen intake, or transference to intensive care unit from ventilator using. All adverse events would be documented and evaluated by an independent committee.

\section{Sample size and statistical analysis}

We defined the minimum improvement rate as the increase of lymphocytes by more than $40 \%$ per 24 hours. Assuming the mean change of lymphocyte counts in the treatment group and the control group was 0.7 and 0.5 respectively, then a total of 24 patients were needed with the power of $80 \%$, with the $5 \%$ probability of type冈error $(a=0.05)$.

Continuous variables are expressed as mean $\pm S D$ and are compared using the independent t-test. Categorical variables are expressed by numbers (percentages) and are compared using the chi-square test 
or Fisher's exact test. A 2-sided P value $<0.05$ was considered significant. All statistical analyses were performed using SPSS version 22.0.

\section{Results}

\section{Baseline characteristics}

From March 1, 2020, to April 2, 2020, a total of 24 patients with the first occurrence of the absolute lymphocyte level $<1.1 * 10^{9} / \mathrm{L}$ during hospitalization were recruited in this randomized controlled trial. 12 patients $(50.0 \%)$ were randomized to receive the routine treatments plus niacinamide, and 12 patients $(50.0 \%)$ were randomized to receive the routine treatments alone (Fig.1). The age of participants in our study ranged from 46 to 91 years old, with the mean age of $69.1 \pm 11.8$ years old. The mean age in the treatment group and control group was $69 \pm 12$ and $70 \pm 12$ years old, respectively ( $P=0.928$, Table 1 ). In our study, 11 participants $(45.83 \%)$ were male, with 7 participants $(58.3 \%)$ in the treatment group and 4 participants $(33.3 \%)$ in the control group $(\mathrm{P}=0.219$, Table 1$) .3$ patients $(25 \%)$ in the treatment group and 2 patients $(16.7 \%)$ in the control group were diagnosed as the ordinary type, with no statistical significance between the two groups ( $P=0.253$, Table 1$)$. Other baseline characteristics were comparable with no statistical significance between the two groups $(P>0.05)$, as is shown in Table 1.

\section{Comparison in laboratory tests}

The median time for patients from hospitalization to enrollment into our study was 1 (1-3.75) days. Before treatment, the overall lymphocyte counts in our study were $(0.91 \pm 0.22) * 10^{9} / \mathrm{L}$ with $(0.94 \pm 0.26) * 10^{9} / \mathrm{L}$ in the treatment group and $(0.89 \pm 0.19) * 10^{9} / \mathrm{L}$ in the control group. There was no statistical significance between the two groups $(P=0.565$, Table 3$)$. Other laboratory parameters, such as leukocyte counts, absolute monocyte counts, absolute lymphocyte counts, and absolute eosinophilic cell counts before and after receiving the intervention were all comparable in the two groups $(P>0.05)$, as shown in Table 2.

\section{Study endpoint}

Based on routine treatments, patients in the intervention group additionally received niacinamide. For the primary endpoint, the lymphocyte counts after intervention in the treatment or control group were $(1.15 \pm 0.48) * 10^{9} / \mathrm{L}$ and $(1.02 \pm 0.28) * 10^{9} / \mathrm{L}$, respectively. The difference was not statistically significant between the two groups $(P=0.445$, Table 3$)$. Moreover, although the lymphocyte improvement rate in the treatment group was higher than the control group (mean $\pm S D$ for the treatment group: $23.08 \pm 46.10 \%$; control group: $16.52 \pm 24.10 \%, P=0.67$ ), there was no statistically significant difference between the two groups $(P>0.05)$, as is shown in Table 3. For the secondary endpoints, no patient died in the hospital within 48 hours after treatment, and there was no statistically significant difference in the composite endpoint of aggravation between the two groups $(P>0.05)$.

\section{Discussion}


In the present study, the efficiency of nicotinamide on peripheral blood lymphocyte decline in COVID-19 patients was analyzed. Among patients with COVID-19, although the lymphocyte improvement rate was higher in the nicotinamide group, there was no significant difference in either the absolute change of lymphocyte counts or the elevation rate of lymphocytes between the intervention group with nicotinamide and the control group with only routine treatments. Besides, other laboratory parameters before and after receiving the intervention were also comparable in the two groups. As a result, we found no evidence that the niacinamide may impose an effect on lymphopenia in patients with COVID-19.

Patients with severe or critically severe COVID-19 often have significantly increased white blood cell (WBC) counts, neutrophil to lymphocyte ratio (NLR), D-dimer, and fibrinogen levels, but have decreased lymphocyte and platelet[3-6]. As a result, these biomarkers are recommended as indicators of the severity of COVID-19 patients[5]. Among these biomarkers, the decrease of the lymphocyte counts is highly related to the progression of COVID-19[13]. Fu et al[6] showed that the lymphocyte counts were higher in the mild group compared with the severe group ( $1.42 \pm 0.66$ vs $0.97 \pm 0.33, P=0.009)$, showing the association between the illness severity and the lymphocyte counts. Moreover, the peripheral lymphocyte subset alteration also showed the characteristics of progression of the severe disease. The CD4+ T cells, CD8+ T cells, B cells, and NK cells all showed a significant reduction in the patients with severe COVID-19 compared to patients with ordinary or mild type[1,7,14] and all these cells were gradually decreasing as the disease progressed gradually. Some studies, therefore, showed the CD4+ T cells, CD8+ T cells, and the CD4+/CD8+ ratios were the independent predictors for the severity of COVID-19[1,14]. Besides, the percentage of interferon- $\gamma$ (IFN- $\gamma$ ) producing CD8+ T cells and IFN- $\gamma$ producing CD4+ T cells both increased in severe COVID-19 patients, which indicated the severity of the disease was associated with the hyperfunction of CD4+ T cells and CD8+ T cells, and the cytokines played a pivotal role in the progression of the disease[15].

As described earlier, the reduction of peripheral T cells and their subsets, as unique characteristics of COVID-19 patients, is closely related to the severity and prognosis of the disease, which has been repeatedly confirmed in clinical observation and autopsy pathological reports. Similar to SARS-CoV and SARS-CoV-2 infections, the lymphopenia in acute COVID-19 patients may be associated with lymphocyte infiltration and sequestration in specific target organs, like lungs[16, 17]. However, in the later stages of COVID-19 disease, lymphopenia is mostly mediated by hyper inflammation and cytokine release[18]. Therefore, we presumed that lymphopenia in COVID-19 patients may be mainly related to the cytokine storm. Cytokine storm refers to an excessive immune response triggered by a sharp increase in the number of pro-inflammatory cytokines, resulting in an abnormal immune response[19, 20]. By analyzing the clinical characteristics of COVID-19 patients, we found that their serum proinflammatory cytokines significantly increased, which indicated the existence of cytokine storm and predicted the severity of COVID-19[4, 5, 21, 22]. A study including 138 hospitalized COVID-19 patients suggested that the death of COVID-19 patients might be related to the cytokine storm induced by the virus[23]. Liu et al[22] also revealed that the severity of lymphocytopenia and proinflammatory cytokine storms were higher in patients with severe COVID-19 than in patients without severe COVID-19 and highly correlated with the severity of the disease. Furthermore, the biopsy samples analysis of 19 patients with COVID-19 undergoing 
autopsy showed abnormal host immune response and inflammatory cytokine storm as well as mononuclear inflammatory lymphocytes in the biopsy specimen. Fu et al[24] thought that the pathogenic T cells and inflammatory monocytes can enter the pulmonary circulation in great quantities, resulting in cytokine storm in patients with severe or critically severe illness. The cytokine storm will gradually increase the alveolar exudate, hamper the alveolar gas exchange, and finally increase the mortality in patients with severe disease. Previous studies also showed that the reduction of the inflammatory cytokine storm opposed lymphopenia[25]. RECOVERY study[26], the largest RCT study which explored the therapeutic effect of tocilizumab (interleukin-6 inhibitors) on patients with COVID-19, demonstrated a significant benefit in 28-day mortality after the intervention. These results confirmed the promising application of modulating and stabilizing cytokine storms for COVID-19 treatment.

Previous studies have found that the increase of many cytokines, such as interleukin-6 (IL-6), interleukin-10 (IL-10), tumor necrosis factor (TNF) -2, IFN-y was the main cause of cytokine storm in vivo[21, 22, 27, 28]. As a key factor of inflammation triggering, IL-6 can be highly expressed in patients with COVID-19, which is associated with the decrease in the lymphocyte counts, inflammation caused by cytokine storm, and severity of disease $[23,28,29]$. So the target drug that inhibits IL- 6 may theoretically block the cytokine storm[29-31].NAD+, derived from the niacinamide, modulate the cytokine action, regulate the intercellular adhesion molecules, block degranulation of mast cells and inhibit protease release from leukocytes[32]. Moreover, the NAD+ is a substrate of PARP which will act as an inflammatory meditator via the activation of nuclear factor-KB (NF-KB)[32]. So the nicotinamide usefully blocks the cytokine storm by modulating inflammatory factors including interleukin-1 $\beta$ (IL-1 $\beta$ ), IL-6, interleukin-8 (IL-8), IL-10, monocyte chemoattractant protein (MCP-1), and TNF-a[33]. Recently, some hypotheses have been put forward that the CD38 and CD38-mediated NAD+ metabolism plays a central role in altered immunometabolism resulting from COVID-19 infection. The CD38/NAD+ axis may become a promising therapeutic target that allows niacinamide therapy to have a brilliant future[34] (Fig.2). Furthermore, recent studies have shown that nicotinamide riboside (NR), the precursor of nicotinamide, has close structural similarity to the proven RNA-dependent RNA polymerase (RdRp) inhibitors which inhibit the proliferation of the viruses[35].

Network pharmacology-based and computational analyses also showed that niacin could help in treating colorectal cancer (CRC) patients infected with COVID-19 through enhancement of immunologic functions, inhibition of inflammatory factors, and regulation of cellular microenvironment[36]. These studies further confirmed the therapeutic effect of nicotinamide in patients with COVID-19. Previous studies have demonstrated that nicotinamide reduced the apoptosis of peripheral blood lymphocytes (PBL) in patients with human immunodeficiency virus (HIV) by mean of protecting mitochondria from the damage of TNFalpha and reactive oxygen species which are mainly induced by cytokine[37, 38]. Likewise, the replenishment of nicotinamide theoretically prevented the absolute counts of lymphocytes from decreasing in the patients with COVID-19. However, whether the nicotinamide-based supportive therapy also increases the lymphocyte counts for patients with COVID-19 remains unclear.

In our study, the absolute lymphocyte counts didn't increase significantly at $48 \mathrm{~h}$ after intervention in both the treatment and control groups. The reason may be that the cytokines leading to the reduction of lymphocytes could not be instantly blocked by niacinamide and there are still other underlying 
mechanisms in the progression of the disease besides cytokine storm. Unfortunately, our study could not evaluate the long-term effect of nicotinamide on lymphopenia for the limited resource during the pandemic. We hope that the therapeutic effect of long-term, even after discharge, administration of niacinamide could be confirmed by more large-scale clinical studies in the future.

\section{Study limitations}

This study has several limitations. Firstly, our study just enrolled 24 COVID-19 patients, which covered a small piece of the population. Although the sample size was calculated in our study to meet our demand, there are still few patients who should be enrolled in. Secondly, we only compared the outcomes between two groups at $48 \mathrm{~h}$ after intervention and didn't evaluate the long-term effect of nicotinamide on lymphopenia for the resource shortage during the pandemic. Moreover, our study adopted the laboratory parameter as our primary endpoint rather than a hard endpoint such as the death mostly because of the inconvenience of eligible patients enrolled.

\section{Conclusions}

In our present study, changes in absolute lymphocyte counts between the niacinamide group and the control group were not significantly different. Therefore, there is currently no evidence in the present study supporting the effect of niacinamide on lymphopenia in patients with COVID-19.

\section{Abbreviations}

COVID-19: Coronavirus disease 2019; SARS-CoV-2: Severe Acute Respiratory Syndrome Corona Virus 2; PARPs: poly-ADP-ribose polymerases; ULN: upper limit of normal; WBC: white blood cell counts; NLR:

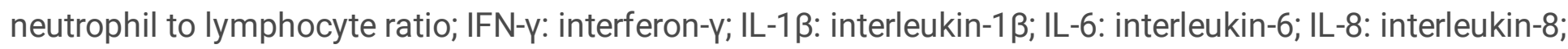
IL-10: interleukin-10; TNF: tumor necrosis factor; NF-KB: nuclear factor-KB; MCP-1: monocyte chemoattractant protein-1; NR: nicotinamide riboside; RdRp: RNA-dependent RNA polymerase; CRC: colorectal cancer; PBL: peripheral blood lymphocytes; HIV: human immunodeficiency virus; COPD: chronic obstructive pulmonary disease; ACEl: angiotensin-converting enzyme inhibitors; ARB: angiotensin receptor blockers; ADPR: adenosine diphosphate ribose; cADPR: cyclic adenosine phosphate ribose; NAADP: nicotinic acid adenine dinucleotide phosphate

\section{Declarations}

\section{Ethical approval}

Our study was approved by the National Health Commission of China, the institutional review board at Huo Shen Shan Hospital, and the institutional ethics committee of the General Hospital of Northern Theater Command of the Chinese People's Liberation Army.

\section{Conflict of interest}


All the authors declare that they have no competing interests.

\section{Consent for publication}

No individual person's data was reported that would require consent to publish from the participant.

\section{Informed consent}

All participants provided written informed consent.

\section{Availability of data and materials}

The data underlying this article will be shared upon reasonable request to the corresponding author.

\section{Funding}

This research was supported by the National Natural Science Foundation of China (NSFC: 32071116 ) and the Lixinyangfan fund (BJUHFCSOARF201901-14). The study funder had no role in the design of the study, the collection, analysis of the data, and in the writing of the paper.

\section{Author's contributions}

QH was responsible for the statistical analysis and wrote the draft; QYZ collected the data and revised this draft; CFP collected the data; ZM collected the data; YLH designed this study. All the authors read and approved the final manuscript.

\section{Acknowledgements}

We would like to thank the staff of the No. 7 Department of Infectious Diseases, Wuhan Huo Shen Shan Hospital, Wuhan Hubei Province.

\section{Reference}

1. Huang W, Berube J, McNamara M, Saksena S, Hartman M, Arshad T, et al. Lymphocyte Subset Counts in COVID-19 Patients: A Meta-Analysis. Cytometry A. 2020;97(8):772-6.

2. Mustafa F, Giles R, Pepper MS. Rapid evolution of our understanding of the pathogenesis of COVID19: Implications for therapy. S Afr Med J. 2020;110(12):1180-5.

3. Ponti G, Maccaferri M, Ruini C, Tomasi A, Ozben T. Biomarkers associated with COVID-19 disease progression. Crit Rev Clin Lab Sci. 2020;57(6):389-99.

4. Akbari $\mathrm{H}$, Tabrizi R, Lankarani KB, Aria H, Vakili S, Asadian F, et al. The role of cytokine profile and lymphocyte subsets in the severity of coronavirus disease 2019 (COVID-19): A systematic review and meta-analysis. Life Sci. 2020;258:118167.

5. Henry BM, de Oliveira MHS, Benoit S, Plebani M, Lippi G. Hematologic, biochemical and immune biomarker abnormalities associated with severe illness and mortality in coronavirus disease 2019 
(COVID-19): a meta-analysis. Clin Chem Lab Med. 2020;58(7):1021-8.

6. Fu J, Kong J, Wang W, Wu M, Yao L, Wang Z, et al. The clinical implication of dynamic neutrophil to lymphocyte ratio and D-dimer in COVID-19: A retrospective study in Suzhou China. Thromb Res. 2020;192:3-8.

7. Zhang W, Li L, Liu J, Chen L, Zhou F, Jin T, et al. The characteristics and predictive role of lymphocyte subsets in COVID-19 patients. Int J Infect Dis. 2020;99:92-9.

8. Tan L, Wang Q, Zhang D, Ding J, Huang Q, Tang YQ, et al. Lymphopenia predicts disease severity of COVID-19: a descriptive and predictive study. Signal Transduct Target Ther. 2020;5(1):33.

9. Bogan-Brown K, Nkrumah-Elie Y, Ishtiaq Y, Redpath P, Shao A. Potential Efficacy of Nutrient Supplements for Treatment or Prevention of COVID-19. J Diet Suppl. 2021:1-29.

10. Rahimmanesh I, Kouhpayeh S, Khanahmad H. The Conceptual Framework for SARS-CoV-2 Related Lymphopenia. Preprints 2020, 2020040045. 2020.

11. Mehmel M, Jovanovic N, Spitz U. Nicotinamide Riboside-The Current State of Research and Therapeutic Uses. Nutrients. 2020;12(6).

12. National Health Commission of the People's Republic of China. Guidelines for The Diagnosis and Treatment of Novel Coronavirus (2019- nCoV) Infection by The National Health Commission (trial version 6) (2020-03-07) [EB/OL].

http://www.nhc.gov.cn/xcs/zhengcwj/202002/8334a8326dd94d329df351d7da8aefc2.shtml

13. Klöppel R, Zöllner H, Wieniecki P, Kamprad F, Oelssner W. [Multivariate discrimination analysis of enzyme patterns in tumor patients]. Radiobiol Radiother (Berl). 1982;23(2):127-33.

14. Zhang Y, Mo P, Ma Z, Song S, Deng L, Xiong Y, et al. Characteristics of Peripheral Lymphocyte Subset Alteration in COVID-19 Pneumonia. The Journal of Infectious Diseases. 2020;221(11):1762-9.

15. Wang F, Hou H, Luo Y, Tang G, Wu S, Huang M, et al. The laboratory tests and host immunity of COVID-19 patients with different severity of illness. JCI Insight. 2020;5(10).

16. Xu Z, Shi L, Wang Y, Zhang J, Huang L, Zhang C, et al. Pathological findings of COVID-19 associated with acute respiratory distress syndrome. The Lancet Respiratory Medicine. 2020;8(4):420-2.

17. T L, Z Q, L Z, Y H, W H, Z L, et al. Significant changes of peripheral T lymphocyte subsets in patients with severe acute respiratory syndrome. 2004;189(4):648-51.

18. R S, L C, A DP, Pediatrics CFJ, neonatology. Role of thymopoiesis and inflamm-aging in COVID-19 phenotype. 2020;61(3):364-5.

19. Channappanavar R, Perlman S. Pathogenic human coronavirus infections: causes and consequences of cytokine storm and immunopathology. Semin Immunopathol. 2017;39(5):529-39.

20. Fajgenbaum DC, June CH. Cytokine Storm. N Engl J Med. 2020;383(23):2255-73.

21. Wang D, Hu B, Hu C, Zhu F, Liu X, Zhang J, et al. Clinical Characteristics of 138 Hospitalized Patients With 2019 Novel Coronavirus-Infected Pneumonia in Wuhan, China. JAMA. 2020;323(11):1061-9.

22. Liu J, Li S, Liu J, Liang B, Wang X, Wang H, et al. Longitudinal characteristics of lymphocyte responses and cytokine profiles in the peripheral blood of SARS-CoV-2 infected patients.

EBioMedicine. 2020;55:102763.

Page $10 / 16$ 
23. Zhu Z, Cai T, Fan L, Lou K, Hua X, Huang Z, et al. Clinical value of immune-inflammatory parameters to assess the severity of coronavirus disease 2019. Int J Infect Dis. 2020;95:332-9.

24. Fu B, Xu X, Wei H. Why tocilizumab could be an effective treatment for severe COVID-19? J Transl Med. 2020;18(1):164.

25. Fathi N, Rezaei N. Lymphopenia in COVID-19: Therapeutic opportunities. Cell Biol Int. 2020;44(9):17927.

26. Lancet RCGJ. Tocilizumab in patients admitted to hospital with COVID-19 (RECOVERY): a randomised, controlled, open-label, platform trial. 2021;397(10285):1637-45.

27. Chen L, Liu HG, Liu W, Liu J, Liu K, Shang J, et al. [Analysis of clinical features of 29 patients with 2019 novel coronavirus pneumonia]. Zhonghua Jie He He Hu Xi Za Zhi. 2020;43(3):203-8.

28. Wang J, Jiang M, Chen X, Montaner LJ. Cytokine storm and leukocyte changes in mild versus severe SARS-CoV-2 infection: Review of 3939 COVID-19 patients in China and emerging pathogenesis and therapy concepts. J Leukoc Biol. 2020;108(1):17-41.

29. Chen X, Zhao B, Qu Y, Chen Y, Xiong J, Feng Y, et al. Detectable Serum Severe Acute Respiratory Syndrome Coronavirus 2 Viral Load (RNAemia) Is Closely Correlated With Drastically Elevated Interleukin 6 Level in Critically III Patients With Coronavirus Disease 2019. Clin Infect Dis. 2020;71(8):1937-42.

30. Tanaka T, Narazaki M, Kishimoto T. Immunotherapeutic implications of IL-6 blockade for cytokine storm. Immunotherapy. 2016;8(8):959-70.

31. Zhang S, Li L, Shen A, Chen Y, Qi Z. Rational Use of Tocilizumab in the Treatment of Novel Coronavirus Pneumonia. Clin Drug Investig. 2020;40(6):511-8.

32. Omran HM, Almaliki MS. Influence of NAD+ as an ageing-related immunomodulator on COVID 19 infection: A hypothesis. J Infect Public Health. 2020;13(9):1196-201.

33. Monfrecola G, Gaudiello F, Cirillo T, Fabbrocini G, Balato A, Lembo S. Nicotinamide downregulates gene expression of interleukin-6, interleukin-10, monocyte chemoattractant protein-1, and tumour necrosis factor-alpha gene expression in $\mathrm{HaCaT}$ keratinocytes after ultraviolet $\mathrm{B}$ irradiation. Clin Exp Dermatol. 2013;38(2):185-8.

34. JD Z, S K, KA H, reviews CEJP. Implications of the NADase CD38 in COVID pathophysiology. 2021.

35. Z E, M A, M L, structure BAJJom. Molecular docking and dynamics studies of Nicotinamide Riboside as a potential multi-target nutraceutical against SARS-CoV-2 entry, replication, and transcription: A new insight. 2022;1247:131394.

36. R L, Y L, X L, L Y, M S, bioinformatics LKJBi. Network Pharmacology and bioinformatics analyses identify intersection genes of niacin and COVID-19 as potential therapeutic targets. 2021;22(2):127990.

37. A C, C M, N M, V B, A S, B DR, et al. Mitochondria alterations and dramatic tendency to undergo apoptosis in peripheral blood lymphocytes during acute HIV syndrome. 1997;11(1):19-26.

38. A S, C M, GC O, C C, L C, PG P, et al. Apoptotic DNA fragmentation, and its in vitro prevention by nicotinamide, in lymphocytes from HIV-1-seropositive patients and in HIV-1-infected MT-4 cells. 
1997;15(3):171-9.

\section{Tables}

Table 1. Baseline characteristics of patients in both groups

\begin{tabular}{|c|c|c|c|c|}
\hline & Treatment $₫ n=12 \rrbracket$ & 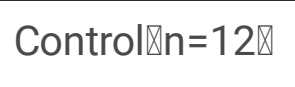 & $x^{2} / t$ & $P$ \\
\hline Men & $7 \rrbracket 58.3 \rrbracket$ & $4 \llbracket 33.3 \rrbracket$ & 1.510 & 0.219 \\
\hline Age(years) & $69 \pm 12$ & $70 \pm 12$ & 0.136 & 0.928 \\
\hline Admission respiratory rate & $23.9 \pm 3.7$ & $23.3 \pm 1.7$ & -0.500 & 0.622 \\
\hline Admission heart rate & $91.0 \pm 10.0$ & $86.5 \pm 13.8$ & -0.914 & 0.370 \\
\hline \multicolumn{5}{|l|}{ Diagnosis } \\
\hline Ordinary, \% & $3 \rrbracket 25.0 \rrbracket$ & 2ه16.7区 & 0.253 & 0.615 \\
\hline Severe, \% & $9 ه 75.0 \rrbracket$ & 10ه83.3》 & 0.253 & 0.615 \\
\hline Hypertension & $2 \otimes 16.7 \rrbracket$ & $6 \rrbracket 50.0 \rrbracket$ & 3.0 & 0.083 \\
\hline Coronary heart disease & $0 \otimes 0.0 \rrbracket$ & $2 \otimes 16.7 \rrbracket$ & 2.182 & 0.140 \\
\hline Diabetes & $1 \rrbracket 8.3 \rrbracket$ & $3 \rrbracket 25.0 \rrbracket$ & 1.200 & 0.273 \\
\hline Cerebrovascular disease & $0 \otimes 0.0 \rrbracket$ & 2ه16.7》 & 2.182 & 0.140 \\
\hline Liver dysfunction & $0 \otimes 0.0 \rrbracket$ & $2 \otimes 16.7 \rrbracket$ & 2.182 & 0.140 \\
\hline Renal insufficiency & $1 \rrbracket 8.3 \rrbracket$ & $0 \otimes 0.0 \bigotimes$ & 1.043 & 0.307 \\
\hline COPD & $0 \otimes 0.0 \rrbracket$ & $0 \otimes 0.0 \rrbracket$ & - & - \\
\hline Antiviral drug, \% & $1 \otimes 100.0 \rrbracket$ & $1 \otimes 100.0 \otimes$ & - & - \\
\hline ACEI/ARB, \% & $0 \rrbracket 0.0 \rrbracket$ & $1 \rrbracket 8.3 \rrbracket$ & 1.043 & 0.307 \\
\hline Calcium antagonist, \% & $1 \rrbracket 8.3 \rrbracket$ & $1 \rrbracket 8.3 \rrbracket$ & 0.000 & 1.000 \\
\hline Hypoglycemic drugs, \% & $1 \rrbracket 8.3 \rrbracket$ & $2 \bowtie 16.7 \rrbracket$ & 0.381 & 0.537 \\
\hline
\end{tabular}

Continuous variables are presented as mean $\pm S D$; categorical variables are expressed by numbers (percentages). COPD, chronic obstructive pulmonary disease; ACEl, angiotensin-converting enzyme inhibitors; ARB, angiotensin receptor blockers

Table 2. Comparison of full blood counts before and after niacinamide taking 


\begin{tabular}{|c|c|c|c|c|c|c|}
\hline & Treatment $\varangle n=1$ & & & 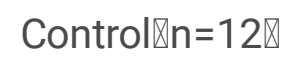 & & \\
\hline & Before & After & $P$ & Before & After & $P$ \\
\hline $\begin{array}{l}\text { leukocyte } \\
\text { counts } \\
\square^{\star} 10^{9} / \mathrm{L} \rrbracket\end{array}$ & $5.50 \pm 1.89$ & $5.33 \pm 1.55$ & 0.710 & $5.41 \pm 1.77$ & $5.03 \pm 1.36$ & 0.484 \\
\hline $\begin{array}{l}\text { absolute } \\
\text { monocyte } \\
\text { counts }\left({ }^{\star} 10^{9} / \mathrm{L} \rrbracket\right.\end{array}$ & $0.41 \pm 0.16$ & $0.38 \pm 0.12$ & 0.270 & $0.38 \pm 0.10$ & $0.37 \pm 0.09$ & 0.832 \\
\hline $\begin{array}{l}\text { absolute } \\
\text { lymphocyte } \\
\text { counts }\left({ }^{*} 10^{9} / \mathrm{L}\right)\end{array}$ & $0.94 \pm 0.26$ & $1.15 \pm 0.48$ & 0.098 & $0.89 \pm 0.19$ & $1.02 \pm 0.28$ & 0.056 \\
\hline $\begin{array}{l}\text { absolute } \\
\text { eosinophilic } \\
\text { cell } \\
\text { counts( }\left(* 10^{9} / \mathrm{L}\right)\end{array}$ & $0.10 \pm 0.04$ & $0.10 \pm 0.08$ & 0.487 & $0.08 \pm 0.06$ & $0.10 \pm 0.06$ & 0.200 \\
\hline $\begin{array}{l}\text { absolute } \\
\text { basophilic cell } \\
\text { counts }\left({ }^{*} 10^{9} / \mathrm{L}\right)\end{array}$ & $0.03 \pm 0.01$ & $0.03 \pm 0.02$ & 0.820 & $0.02 \pm 0.01$ & $0.02 \pm 0.01$ & 0.339 \\
\hline $\begin{array}{l}\text { absolute } \\
\text { neutrophils } \\
\text { counts }\left(* 10^{9} / \mathrm{L}\right)\end{array}$ & $3.80 \pm 1.74$ & $3.75 \pm 1.58$ & 0.904 & $3.92 \pm 1.86$ & $3.51 \pm 1.15$ & 0.364 \\
\hline $\begin{array}{l}\text { monocytes } \\
\text { percentage, \% }\end{array}$ & $7.43 \pm 1.49$ & $7.53 \pm 2.83$ & 0.887 & $7.45 \pm 2.57$ & $7.46 \pm 1.74$ & 0.989 \\
\hline $\begin{array}{l}\text { lymphocyte } \\
\text { percentage, \% }\end{array}$ & $23.56 \pm 10.25$ & $21.13 \pm 9.77$ & 0.273 & $20.18 \pm 7.83$ & $23.18 \pm 7.46$ & 0.079 \\
\hline $\begin{array}{l}\text { eosinophilic } \\
\text { cell } \\
\text { percentage, \% }\end{array}$ & $1.87 \pm 0.69$ & $1.75 \pm 1.03$ & 0.734 & $1.62 \pm 1.20$ & $2.06 \pm 1.11$ & 0.200 \\
\hline $\begin{array}{l}\text { basophilic cell } \\
\text { percentage, \% }\end{array}$ & $0.63 \pm 0.42$ & $0.56 \pm 0.37$ & 0.597 & $0.35 \pm 0.21$ & $0.42 \pm 0.23$ & 0.339 \\
\hline $\begin{array}{l}\text { neutrophil } \\
\text { percentage, \% }\end{array}$ & $66.52 \pm 10.31$ & $68.87 \pm 10.69$ & 0.433 & $70.40 \pm 9.63$ & $66.88 \pm 8.45$ & 0.148 \\
\hline $\begin{array}{l}\text { red blood cell } \\
\text { counts } \\
\left({ }^{\star} 10^{12} / \mathrm{L}\right)\end{array}$ & $14.10 \pm 25.45$ & $15.64 \pm 25.80$ & 0.492 & $17.06 \pm 20.39$ & $8.29 \pm 11.33$ & 0.371 \\
\hline $\begin{array}{l}\text { platelet counts } \\
\left({ }^{*} 10^{9} / \mathrm{L}\right)\end{array}$ & $195.83 \pm 80.79$ & $185.58 \pm 77.31$ & 0.127 & $167.17 \pm 62.15$ & $167.75 \pm 55.90$ & 0.860 \\
\hline $\begin{array}{l}\text { Hemoglobin } \\
(\mathrm{g} / \mathrm{L})\end{array}$ & $109.42 \pm 14.43$ & $107.25 \pm 18.18$ & 0.552 & $108.00 \pm 15.11$ & $99.58 \pm 31.63$ & 0.417 \\
\hline $\begin{array}{l}\text { C-reactive } \\
\text { protein (mg/L) }\end{array}$ & $14.10 \pm 25.45$ & $15.64 \pm 25.80$ & 0.760 & $17.06 \pm 20.39$ & $8.23 \pm 11.33$ & 0.160 \\
\hline
\end{tabular}


Continuous variables are presented as mean \pm SD

Table 3. absolute lymphocyte counts before and after receiving niacinamide

\begin{tabular}{|lllll|}
\hline Group & Before $\left({ }^{*} 10^{9} / \mathrm{L}\right)$ & After $\left({ }^{*} 10^{9} / \mathrm{L}\right)$ & $\mathrm{P}$ & lymphocyte improvement rate, $\%$ \\
\hline Treatment & $0.94 \pm 0.26$ & $1.15 \pm 0.48$ & 0.098 & $23.08 \pm 46.10$ \\
\hline Control & $0.89 \pm 0.19$ & $1.02 \pm 0.28$ & 0.056 & $16.52 \pm 24.10$ \\
\hline$P$ & 0.565 & 0.445 & & 0.67 \\
\hline
\end{tabular}

Continuous variables are presented as mean $\pm S D$

Figures 


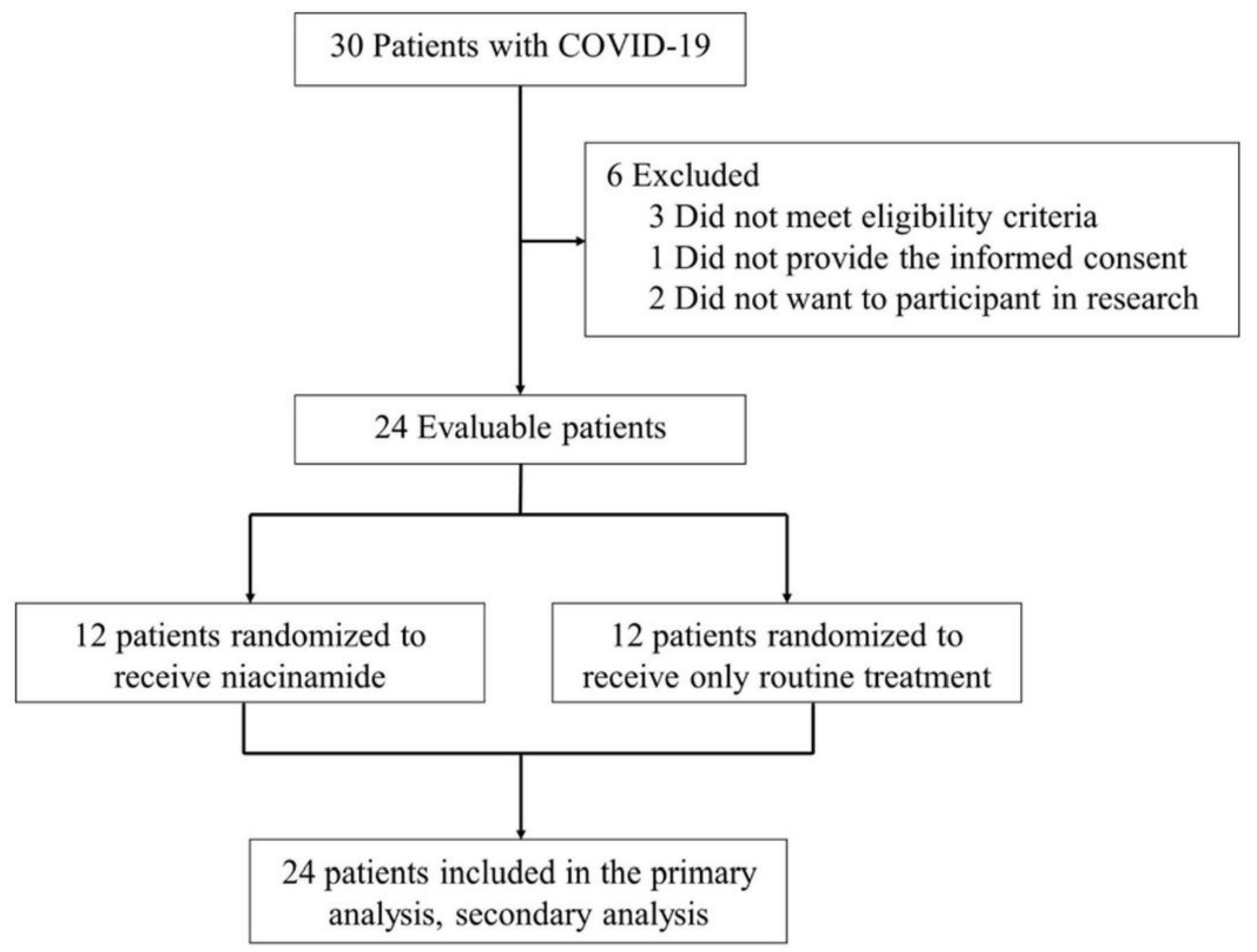

Figure 1

Flow diagram of the study 


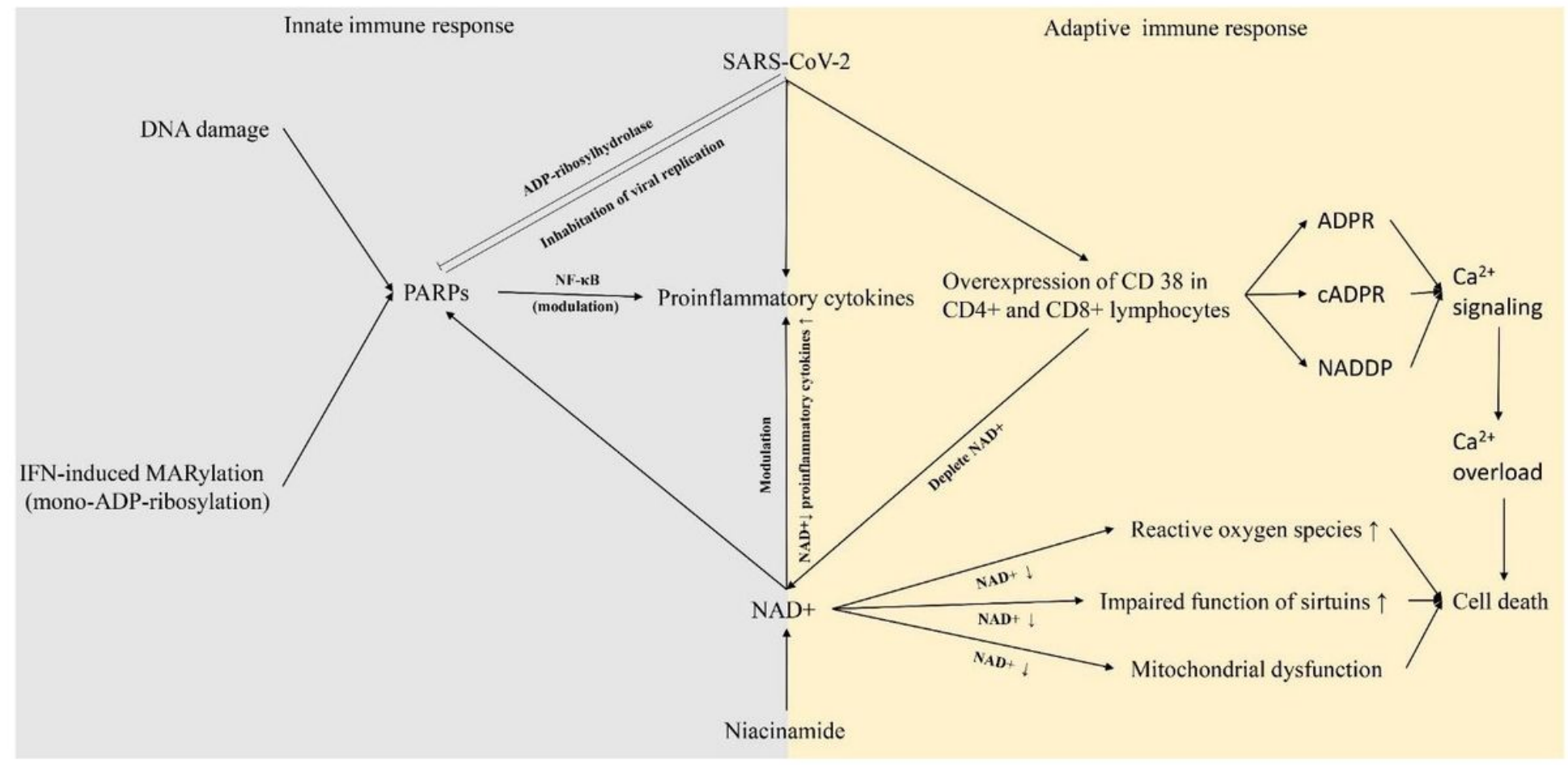

\section{Figure 2}

The immune response in patients with COVID-19.

SARS-CoV-2: Severe Acute Respiratory Syndrome Corona Virus 2; PARPs: poly-ADP-ribose polymerases; NF-KB: nuclear factor-KB; ADPR: adenosine diphosphate ribose; CADPR: cyclic adenosine phosphate ribose; NAADP: nicotinic acid adenine dinucleotide phosphate 